\title{
Beyond Action Recognition: Action Completion in RGB-D Data
}

\section{Farnoosh Heidarivincheh}

farnoosh.heidarivincheh@bristol.ac.uk

Majid Mirmehdi

majid@cs.bris.ac.uk

Dima Damen

dima.damen@bristol.ac.uk
Department of Computer Science

University of Bristol

Bristol, UK
Robust motion representations for action recognition have achieved remarkable performance in both controlled and 'in-the-wild' scenarios. Such representations are primarily assessed for their ability to label a sequence according to some predefined action classes (e.g. walk, wave, open). Although increasingly accurate, these classifiers are likely to label a sequence, even if the action has not been fully completed, because the motion observed is similar enough to the training set. Consider the case where one attempts to drink but realises the beverage is too hot. A drinking-vs-all classifier is likely to recognise this action as drinking regardless. We introduce the term action completion as a step beyond the task of action recognition. It aims to recognise whether the action's goal has been successfully achieved. The notion of completion differs per action and could be infeasible to verify using a visual sensor, however, for many actions, an observer would be able to make the distinction by noticing subtle differences in motion.

We address incompletion in a supervised approach, using a new dataset that contains 414 complete as well as incomplete sequences, captured using a depth sensor, spanning 6 actions (switch, plug, open, pull, pick and drink). For each action, we varied the conditions so the action cannot be completed. For example, for plug, subjects were given a plug that does not match the socket, while for pull, a drawer was locked so could not be pulled, and similarly for the other actions. Given labelled complete and incomplete sequences of the same action, we build a model of completion of that action as a binary classifier for each of our actions.

Since the notion of completion differs per action, a general action completion method should investigate the performance of different types of features to accommodate the various action classes. For example, for the action pick, the difference between complete and incomplete actions originates from the subtle change in body pose when holding an object, or by observing an object in the hand. On the other hand, for the action drink, the speed at which the action is performed is better able to assess the completion. We propose a method that chooses the feature(s) suitable for recognising completion from a pool of depth features using 'leave-oneperson-out' cross validation on the training set and automatically selecting the most discriminative feature(s).

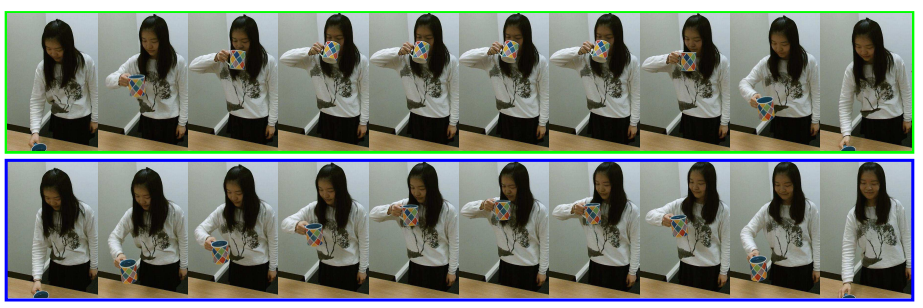

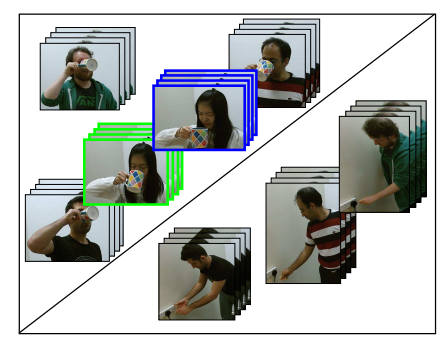

(a) drink vs. plug

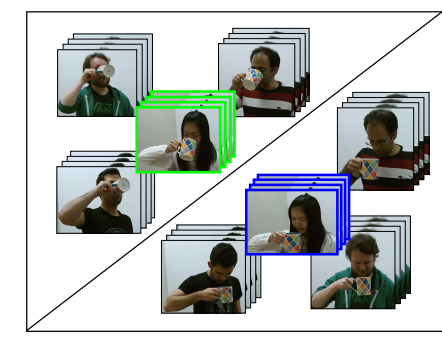

(b) complete vs. incomplete (of drink)
Figure 1: For a complete drink (green) and an incomplete drink (blue) sequences from our dataset, both are classified as drink when using drink vs. plug classifier (a). The proposed supervised action completion model (b) identifies the incomplete sequence.
We present results on a pool of five features: Local Occupancy Pattern (LOP), Joint Positions (JP), Joint Relative Positions (JRP), Joint Relative Angles (JRA) and Joint Velocities (JV) encoded by the Fourier temporal pyramid [1]. On a sequence of experiments, we show that:

1. Complete Action Recognition - The various depth features produce high and comparable \% accuracy for action recognition on our dataset.

2. Incomplete Action Recognition - These features, originally designed for action recognition, behave differently on incomplete action sequences with only some able to distinguish the subtle changes between complete and incomplete sequences of an action.

3. Complete vs. Incomplete Action Recognition - A binary classification was performed as complete vs. incomplete of the same action for each feature. Table 1 shows varying success rates of the different features for the tested actions.

4. Automatic Feature Selection - Using cross validation on training data, the features with the maximum accuracy were selected to build the completion model. By automatic feature selection, we achieve $95.7 \%$ accuracy for recognising action completion across the whole dataset - Table 2.

[1] J. Wang, Z. Liu, Y. Wu, and J. Yuan. Mining actionlet ensemble for action recognition with depth cameras. In CVPR, 2012.

\begin{tabular}{|c|c|c|c|c|c|}
\cline { 2 - 6 } \multicolumn{1}{c|}{} & LOP & JP & JRP & JRA & JV \\
\hline switch & $\mathbf{1 0 0}$ & 85.1 & 85.1 & $\mathbf{1 0 0}$ & $\mathbf{1 0 0}$ \\
\hline plug & 83.6 & 87.7 & 78.1 & 79.5 & $\mathbf{9 4 . 5}$ \\
\hline open & $\mathbf{9 7 . 1}$ & 95.6 & $\mathbf{9 7 . 1}$ & 95.6 & $\mathbf{9 7 . 1}$ \\
\hline pull & 87.3 & 71.8 & 77.5 & 88.7 & $\mathbf{9 4 . 4}$ \\
\hline pick & 92.8 & 94.2 & $\mathbf{9 8 . 6}$ & $\mathbf{9 8 . 6}$ & 95.7 \\
\hline drink & 97 & 97 & 97 & 97 & $\mathbf{1 0 0}$ \\
\hline
\end{tabular}

Table 1: Complete vs. incomplete action results.

\begin{tabular}{|c|c|c|c|c|c|c|c|c|c|}
\hline & \multicolumn{9}{|c|}{ Subjects } \\
\hline & 1 & 2 & 3 & 4 & 5 & 6 & 7 & 8 & total \\
\hline switch & 100 & 100 & 100 & 100 & 100 & 100 & 100 & 100 & 100 \\
\hline plug & 83.3 & 100 & 87.5 & 100 & 88.9 & 100 & 100 & 100 & 94.5 \\
\hline open & 100 & 85.7 & 100 & 100 & 100 & 87.5 & 90 & 100 & 95.6 \\
\hline pull & 88.9 & 100 & 100 & 100 & 100 & 87.5 & 80 & 100 & 94.4 \\
\hline pick & 90 & 100 & 100 & 100 & 100 & 100 & 50 & 100 & 92.8 \\
\hline drink & 77.8 & 100 & 100 & 100 & 100 & 100 & 100 & 100 & 97 \\
\hline & & & & & & & & total & 95.7 \\
\hline
\end{tabular}

Table 2: General action completion results (a) label:
complete switch
predicted: complete switch

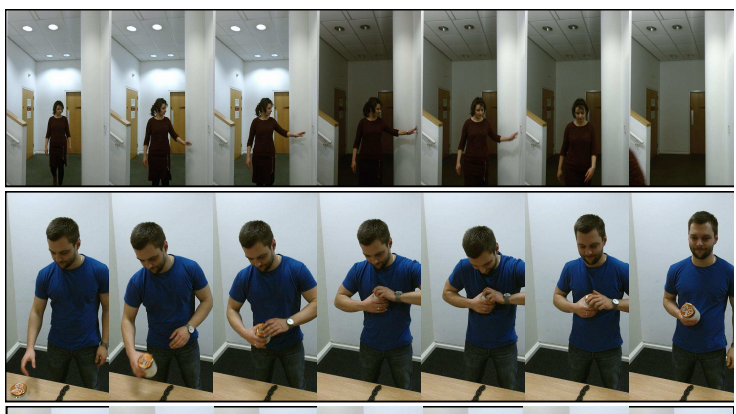

(c) label: incomplete pull predicted: complete pull

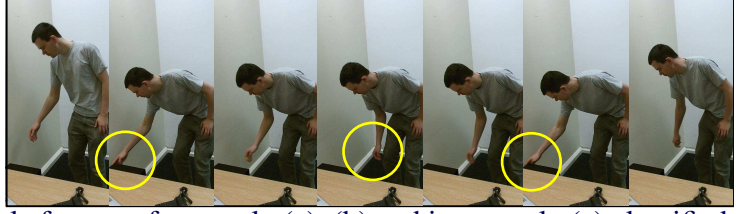

Figure 2: Sample frames of correctly (a), (b) and incorrectly (c) classified test sequences. In (c), using JV solely, the hand seems to perform a pull in full even when the drawer remains unmoved. 